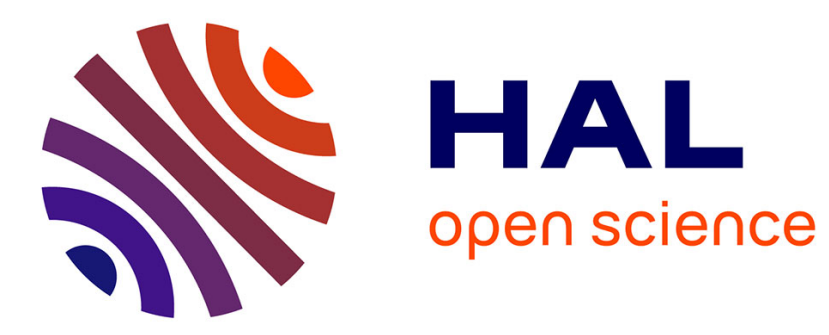

\title{
Demonstrators and multifunctional testbeds for organic semiconductor characterization
}

\author{
L. Fortuna, M. La Rosa, D. Nicolosi, G. Sicurella, E. Umana
}

\section{To cite this version:}

L. Fortuna, M. La Rosa, D. Nicolosi, G. Sicurella, E. Umana. Demonstrators and multifunctional testbeds for organic semiconductor characterization. European Physical Journal: Applied Physics, 2009, 46 (1), pp.1-3. 10.1051/epjap/2009018 . hal-00480148

\section{HAL Id: hal-00480148 \\ https://hal.science/hal-00480148}

Submitted on 3 May 2010

HAL is a multi-disciplinary open access archive for the deposit and dissemination of scientific research documents, whether they are published or not. The documents may come from teaching and research institutions in France or abroad, or from public or private research centers.
L'archive ouverte pluridisciplinaire HAL, est destinée au dépôt et à la diffusion de documents scientifiques de niveau recherche, publiés ou non, émanant des établissements d'enseignement et de recherche français ou étrangers, des laboratoires publics ou privés. 
EPJ manuscript No.

(will be inserted by the editor)

\title{
Demonstrators and multifunctional testbeds for organic semiconductor characterization
}

\author{
Luigi Fortuna ${ }^{1}$, Manuela La Rosa ${ }^{2}$, Donata Nicolosi ${ }^{1,2}$, Giovanni Sicurella ${ }^{1,2}$, and Elena Umana ${ }^{1}$. \\ 1 Dipartimento di Ingegneria Elettrica Elettronica e dei Sistemi, Universitá degli studi di Catania, viale A. Doria 6, 95125 \\ Catania, Italy, Phone: +39.095.7382327, Fax: +39.095.330793 e-mail: umana@diees.unict.it \\ 2 Post Silicon Technologies Group, Technology R\&D, STMicroelectronics, Stradale Primosole, 50, 95121 Catania, Italy
}

Received: date / Revised version: date

\begin{abstract}
A complete framework for characterization and modelling of organic semiconductor materials is here reported. Multifunctional testbeds have been developed to analyze different organic semiconductors through hybrid demonstrators.
\end{abstract}

PACS. 72.80 Le Polymers; organic Compounds (including organic semiconductors) - 73.61 Ph Polymers; organic Compounds

\section{Introduction}

Organic technology has become an important research topic which involves electronic engineers and material science experts towards the development of innovative electronic applications. In the post-silicon era, organic electronics has been exploited to take advantage of low cost device processing techniques, in terms of reduced manufacturing and wafer costs. The development of a complete organic technology platform represents a fundamental step for organic electronics growth and the realization of organic applications. The basic elements which define the organic technology platform are: organic materials, manufacturing process techniques and the CAD framework [1]. The fundamental element of organic electronics is the organic thin-film transistor (OTFT) developed by different organic materials: conductor, dielectric and semiconductor. Therefore, materials characterization is an important step towards the realization of organic technology devices. In this work, a testing platform has been designed. The behavior of polymer semiconductors has been analyzed through the characterization of single devices such as transistors or logic gates. Moreover, multifunctional testbeds architectures have been designed following the IEEE Standard 1620-2004 "Characterization of Organic Transistors and Materials". Hybrid organic structures have been adopted to obtain a standard semiconductor polymers benchmarking. The characterization of the organic semiconductors and the related AC/DC model OTFT have been performed and device parameters extracted [2].

In section 2 the testing platform design flow is reported. The testbed architecture is described in section 3 by illustrating methodologies for organic transistor modelling. Section 4 draws the conclusions.

\section{Testing Platform}

The adopted testing platforms for organic electronics are hardware/software systems composed by application demonstrators and testbed. The design processes of demonstrator and testbed follow a sequence of steps according to the application definition as reported in Fig. 1.

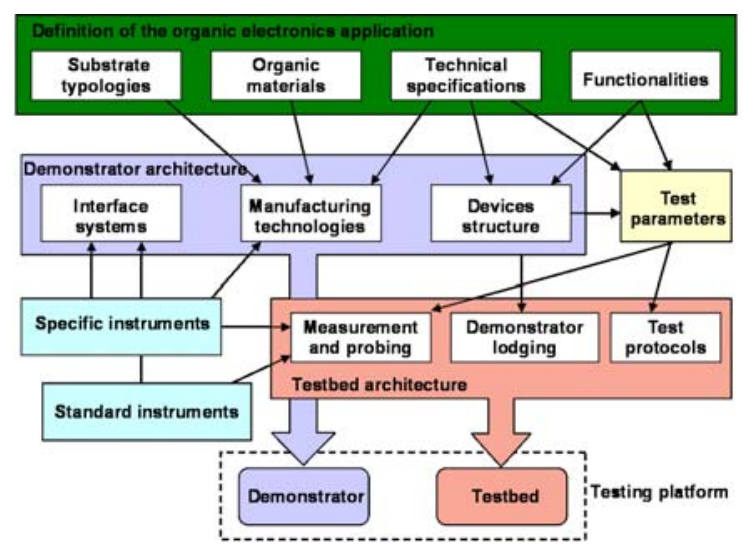

Fig. 1. Testing platform design flow.

The application description, by identifying its characterizing elements and its main functionalities, is the first step of the testing platform design. The choice of the application architecture is heavily affected by materials manufacturing techniques. Organic material deposition, synthesis and patterning impose technological limits to be considered during test platform design. Moreover, every manufacturing technique needs exploiting specifics equipments which influence designing choices. In this phase 
technological data (materials, substrate typologies, etc.), operative information (functionalities) and general technical specifics (processing techniques, feature sizes, etc.) are defined. In the next step ad hoc demonstrators are developed with relative interface systems.

In this work, demonstrators based on metallic contacts with a silicon substrate have been used to obtain templates for organic semiconductors testing. Used demonstrator architectures are thin film transistor (Fig. 2(a)) and logic gate(Fig. 2(b)) with different feature sizes.

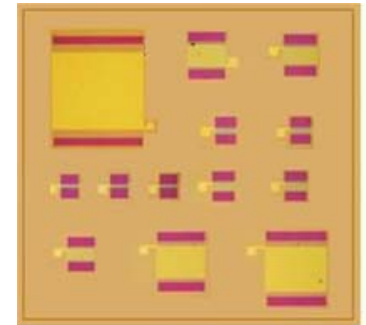

(a)

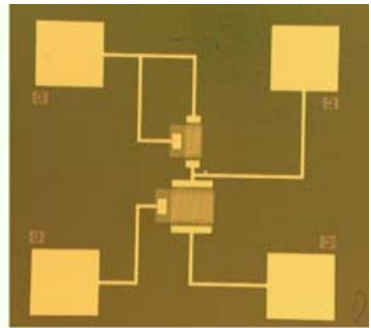

(b)
Fig. 2. Template of pseudo-pmos organic TFT(a) and inverter(b).

In order to investigate the device behavior different geometrical parameters (such as the channel length and the gate dielectric thickness) have been considered. These structures allow the deposition of different organic semiconductor to be tested. Pads and via interconnections have been designed for guaranteeing the electrical contacts with the characterization equipment by means of a probing system. Logic gates have been designed adopting a conventional pseudo-pmos architecture which uses only p-type organic transistors. These configurations are mainly constituted by a driver circuit implementing the logic function and a load circuit usually constituted by a transistor operating in the saturation region. Once the application architecture design is completely developed, its validation becomes essential; for this reason it is necessary the development of multifunctional testbeds. Testbeds are software/hardware systems able to interact with demonstrator samples, designed to implement a set of test protocols. Testbed architecture design can be accomplished through the identification of a set of test parameters. Three main components define the testbed architecture: test protocols, measurement/probing instruments and data lodging systems.

\section{Testbed Architecture}

The testing parameters have been defined through the specification of different measurements. The gate leakage curves $\left(\boldsymbol{I}_{G S}\right.$ vs. $\boldsymbol{V}_{G S}$ and $\boldsymbol{I}_{G D}$ vs. $\left.\boldsymbol{V}_{G D}\right)$ characterize the gate dielectric quality and quantify leakage current from the gate to the channel. Leakage measurements are carried out to ensure gate dielectric integrity before subsequent measurements are performed. The transfer curves $\left(\boldsymbol{I}_{D S}\right.$ vs. $\left.\boldsymbol{V}_{G S}\right)$ allow the preliminary determination of fieldeffect mobility $(\mu)$ and threshold voltage $\left(\boldsymbol{V}_{T}\right)$. The output curves $\left(\boldsymbol{I}_{D S}\right.$ vs. $\left.\boldsymbol{V}_{D S}\right)$ provide saturation and OTFT electrical performance information and are used to determine whether the device exhibits FET-like behaviour. The capacitance curves ( $\boldsymbol{C}_{G D}$ vs. $\boldsymbol{V}_{G S}$ and $\boldsymbol{C}_{G S}$ vs. $\boldsymbol{V}_{G S}$ ) are obtained by the sum of two capacitive contributes: the gate to channel capacitance and the stray capacitance. The stray capacitance values (due to geometrical overlapping of drain source contacts with gate plate) have a negative effect on device switching speed and may affect device electrical characterization. In order to obtain the required set of mesurements, a dedicated multifunctional system, comprising probing, measuring and lodging instruments, has been implemented.

\subsection{DC parameters of OTFT compact model}

A specific strategy has been defined to extract OTFT parameters using electrical measurements. A compact model of organic transistors and a complete characterization of the organic semiconductor have been obtained. The method consists of a differential analysis of the transfer characteristic curves $\left(\boldsymbol{I}_{D}\right.$ vs $\left.\boldsymbol{V}_{G S}\right)$ and exploits the different functional dependences of current on gate voltage which is induced by the presence of contact resistances. Using the expression of drain current in the linear regime:

$$
I_{D}=K\left(V_{G S}-V_{T}\right)^{\gamma+1} V_{D S}
$$

and taking into account the effects of $\boldsymbol{R}_{S}$ and $\boldsymbol{R}_{D}$, letting $\boldsymbol{V}_{G S}=\boldsymbol{V}_{G}$, it is obtained the following quantity which does not depend on $\boldsymbol{R}_{S D}$ :

$$
\begin{gathered}
z=\frac{I_{D}^{2}}{I_{D}^{\prime}}=\frac{K}{\gamma+1}\left(V_{G}-V_{T}\right)^{\gamma+2} V_{D} \\
w=\frac{\int_{V_{T}}^{V_{G}} z d V_{G}^{\prime}}{z} \cong \frac{\int_{0}^{V_{G}} z d V_{G}^{\prime}}{z}=\frac{1}{\gamma+3}\left(V_{G}-V_{T}\right)
\end{gathered}
$$

The threshold voltage $\boldsymbol{V}_{T}$, the mobility parameter $\boldsymbol{K}$ and the source-drain resistance $\boldsymbol{R}_{S D}$ can be extracted by using the quantities $\boldsymbol{z}$ and $\boldsymbol{w}[3]$.

Table 1. Paramater values obtained through used method.

\begin{tabular}{cccc}
\hline$V_{T}$ & $\gamma$ & $R_{S D}$ & $K$ \\
\hline$-1.2 V$ & 0.613 & $1.5 M \Omega$ & $-8.76 e-9 F / V s$ \\
\hline
\end{tabular}

The parameter values obtained by transfer curves have been employed in the compact model implemented in CAD environment. Simulated OTFT behaviours have been compared with experimental data as reported in Fig. 3. 

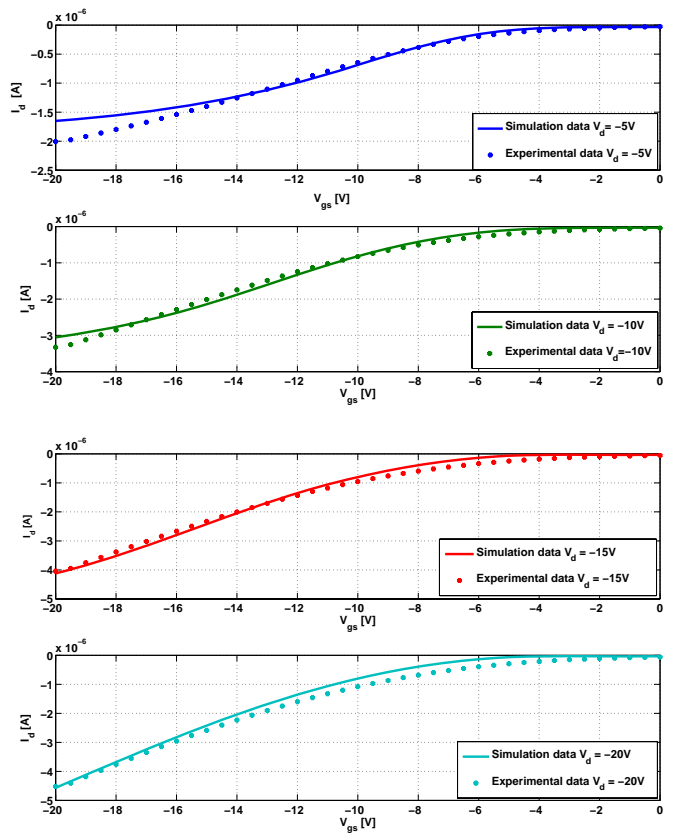

Fig. 3. Experimental and simulation data of OTFT.

\subsection{AC parameters of OTFT compact model}

Dedicated methods have been implemented to extract capacitance values through experimental data fitting. Fig. 4 represents experimental data of device impedance.
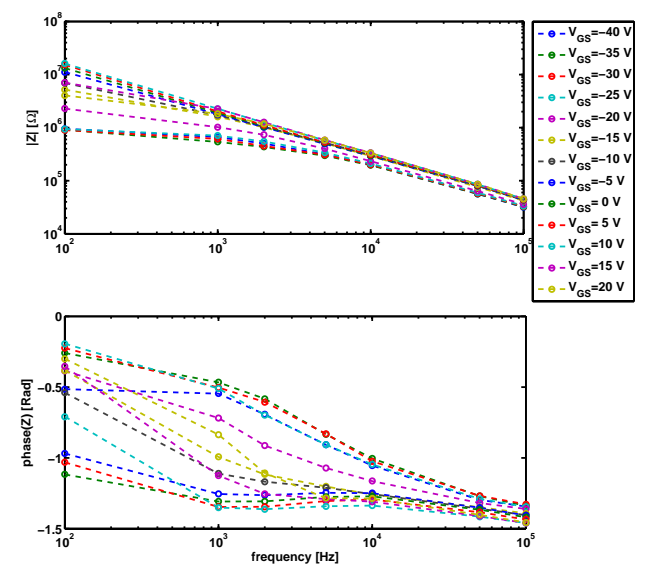

Fig. 4. Measurements of impedance phase and module.

From the impedance analysis of experimental data different behaviours of gate-drain and gate-source impedances have been obtained. Moreover, a parametric study, using different impedance models (two elements RC parallel, three elements R series-RC parallel, four elements with Warburg impedance) has been carried out [4]. Results have been used to validate device impedance behavior with different polarization conditions. In particular, experimental conductance and capacitance mostly showed high variability respect to frequencies variations. Circuit analysis has been performed using the impedance frequency response characteristics in terms of both Bode and Nyquist analysis.

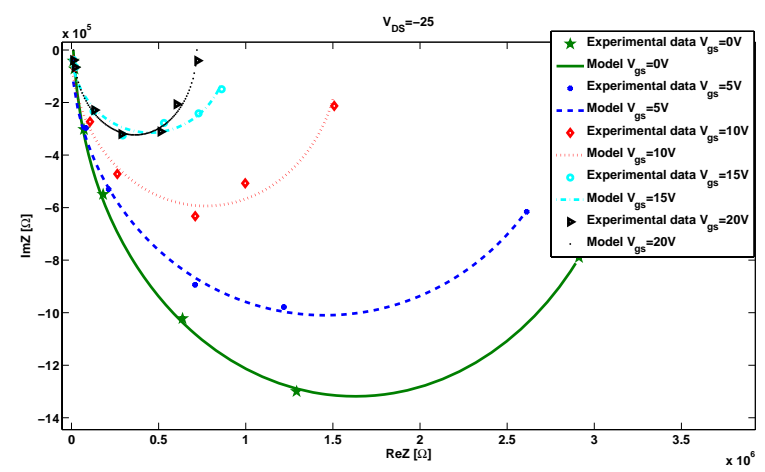

Fig. 5. Measured impedence and equivalent model fitting.

Equivalent impedance model, with relative characteristic parameters, have been identified by fitting simulated Nyquist plot with experimental data (Fig. 5).

\section{Conclusions}

The aim of this work is to develop a complete organic testing platform for organic device validation. A framework for material characterization and OTFT modelling in CAD environment has been designed and validated by experimental data. Demonstrators and testbeds have been implemented and validated.

The authors thank the University of Würzburg for its collaboration in the realization of devices demonstrators.

\section{References}

1. L. Fortuna, M. Frasca, M. La Rosa, G. Sicurella, E. Umana, International Journal of Bifurcation and Chaos 17, (2007) 3035 - 3045.

2. M. La Rosa, D. Nicolosi, L. Occhipinti, G. Sicurella,L. Fortuna, E. Umana, ICOE 08, Eindhoven, (2008).

3. D. Natali, L. Fumagalli, M. Sampietro, Journal of applied physics 101, (2007) 014501.

4. Gabrielli Claude, Solartron Part - Issue B: April 1997, (1997)12860013. 